\title{
Laparoscopic excision of a cystic retroperitoneal adenocarcinoma arising in a focus of extra-ovarian endometriosis
}

\author{
Darren Beiko, MD, FRCSC, DABU, $;^{*}$ S.C. Peter Bryson, MD, FRCSC ; ${ }^{\dagger}$ C. David Bell, MD, FRCPC; ${ }^{+}$ \\ Kenneth T. Pace, MD, MSc, FRCSC ${ }^{\S}$
}

\begin{abstract}
We report an unusual case of a retroperitoneal cystic adenocarcinoma arising in a focus of extra-ovarian retroperitoneal endometriosis. The lesion was completely excised using a transperitoneal laparoscopic approach without the need for any adjuvant chemotherapy or radiation therapy. The patient remains clinically well with no evidence of recurrent or metastatic disease after 3 years of follow-up. To our knowledge, this is the first reported case of this type of rare extra-gonadal endometriosis-associated neoplasm with successful laparoscopic excision.
\end{abstract}

Can Urol Assoc J 2010;4(3):E71-E73

\section{Introduction}

Extra-ovarian endometriosis-associated neoplasms are relatively uncommon and do not typically contain foci of adenocarcinoma. ${ }^{1,2}$ We report an interesting and rare case involving successful laparoscopic surgical monotherapy for a cystic retroperitoneal tumour containing adenocarcinoma that arose in a focus of extra-ovarian endometriosis.

\section{Case report}

An otherwise healthy GOP0 30-year-old female presented with a 1-week history of episodic right upper quadrant and flank pain. There was no significant past gynecologic or urologic history and medications included salbutamol and medroxyprogesterone acetate for depot intramuscular injection. On physical examination, she appeared well and her vital signs were normal. Abdominal examination revealed a scaphoid abdomen with a palpable tender mass in the rightlower quadrant but no peritoneal signs. Pelvic examination was normal. Bloodwork was normal other than elevated liver function enzymes. Transabdominal ultrasound (Fig. 1) and computed tomography scan (Fig. 2) showed a rightsided $6 \times 6 \times 5.5 \mathrm{~cm}$ retroperitoneal cystic lesion unrelated to the kidney, ascending colon or ovary. The mass was fairly well-circumscribed, homogenous, thick-walled and predominantly isoattenuating. Staging was negative. A differential diagnosis of peritoneal inclusion cyst, extragonadal endometriosis, primary retroperitoneal cyst or cystic neoplasm was considered. Given the patient's symptoms, size and appearance of the mass, a cystic malignancy could not be ruled out and informed consent was obtained for laparoscopic excision of the retroperitoneal tumour mass.

The tumour was removed using a 3-trocar, transperitoneal laparoscopic approach with the patient in the left lateral decubitus position. After mobilizing the large intestine and entering the retroperitoneum, we readily identified the cystic mass immediately anterior to the iliopsoas muscles and completely separate from the lower pole of the kidney, ureter, ovary and gonadal vessels. The tumour was carefully dissected free of these structures, placed into an intracorporeal laparoscopic sac where its contents were aspirated. Approximately $100 \mathrm{~mL}$ of dark brown fluid was aspirated and sent to the laboratory for cytology and culture. There was no spillage and the cystic tumour was extracted intact in the sac through a 10 -mm port. Operative time was 95 minutes and there were no intraoperative complications or postoperative complications. The patient was discharged home on the first postoperative day and resumed work by postoperative day 13 .

Gross pathological examination revealed a thick-walled cystic tumour. Histological examination revealed a benign lining of the endometriotic cyst (Fig. 3a) and a moderately well-differentiated adenocarcinoma showing papillary configuration in areas, suggestive of adenocarcinoma arising in a focus of extra-ovarian endometriosis (Fig. 3b). Fluid culture and fluid cytology were negative. After 3 years of follow-up, she remains well with no clinical or radiographic evidence of local or distant disease recurrence.

\section{Discussion}

Endometriosis is a common condition. Although the exact prevalence varies between different patient populations, its prevalence is estimated to be about $10 \%{ }^{3}$ Endometriosis- 


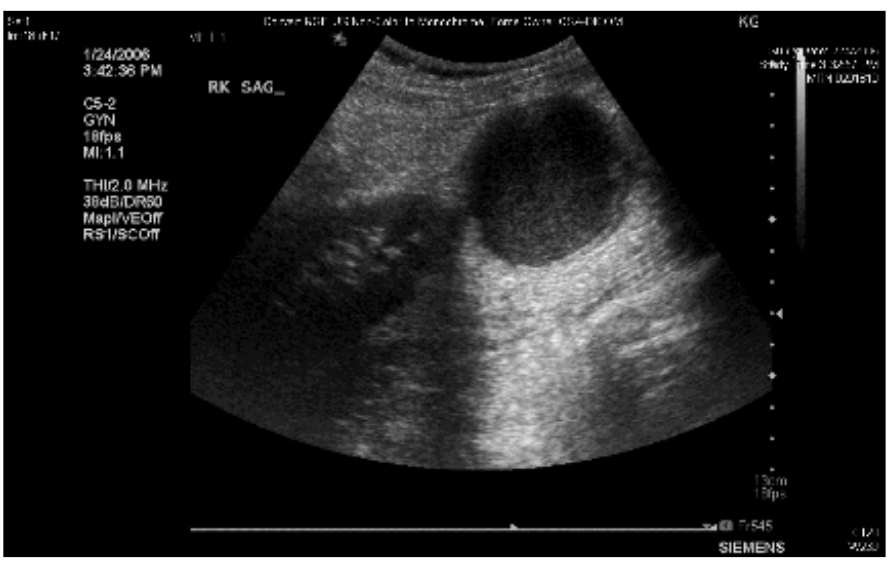

Fig. 1. Ultrasound showing retroperitoneal cyst in relation to the lower pole of the right kidney.

associated neoplasms will develop in $0.5 \%$ to $1 \%$ of women with endometriosis. ${ }^{4,5}$ Extra-gonadal tissues are involved in $25 \%$ to $37 \%$ of these neoplasms, ${ }^{4,6}$ including vagina, fallopian tube or mesosalpinx, pelvic sidewall, colon and parametrium. ${ }^{6}$ Extra-gonadal disease is commonly associated with unopposed estrogen replacement therapy. ${ }^{6,7}$

First-line treatment for endometriosis-associated malignancies is usually surgery, but multimodal therapy is often required, including second-line therapies, such as chemotherapy, radiation therapy and/or hormonal therapy. ${ }^{5}$ The prognosis varies, with 5 -year survival ranging between $10 \%$ to $100 \%$, depending on histologic type and stage at presentation. ${ }^{5}$ Histologic subtypes reported to date in extra-ovarian endometriosis-associated neoplasms include endometrioid carcinoma, endometrial stromal sarcoma and sex cord-stromal tumour with annular tubules. ${ }^{1,2}$

Our patient presented with abdominal pain, which is the presenting symptom in most patients with extra-ovarian endometriosis-associated neoplasms. ${ }^{5}$ Owing to the indeter-

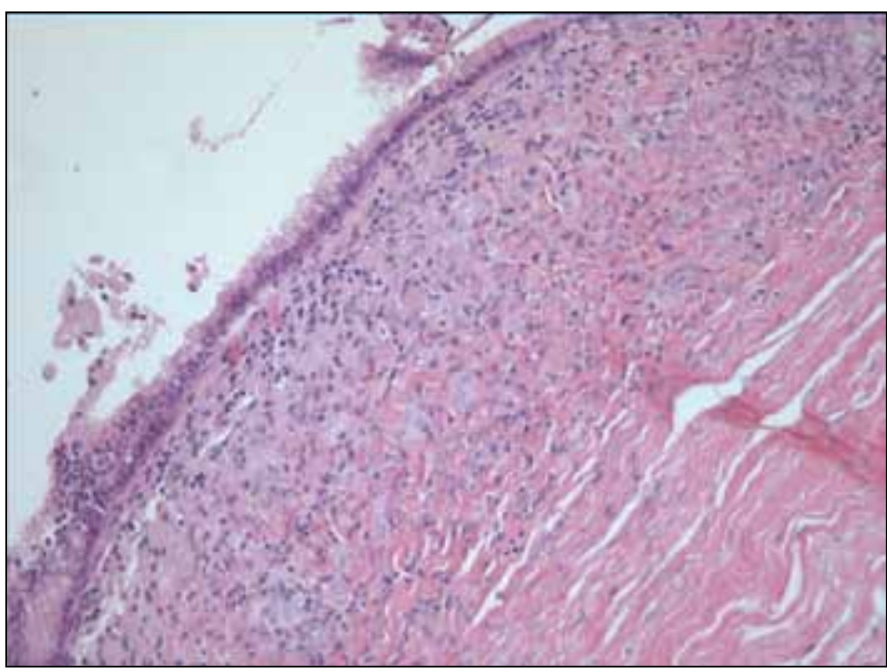

Fig. 3a. Histology showing benign lining of retroperitoneal cyst wall.

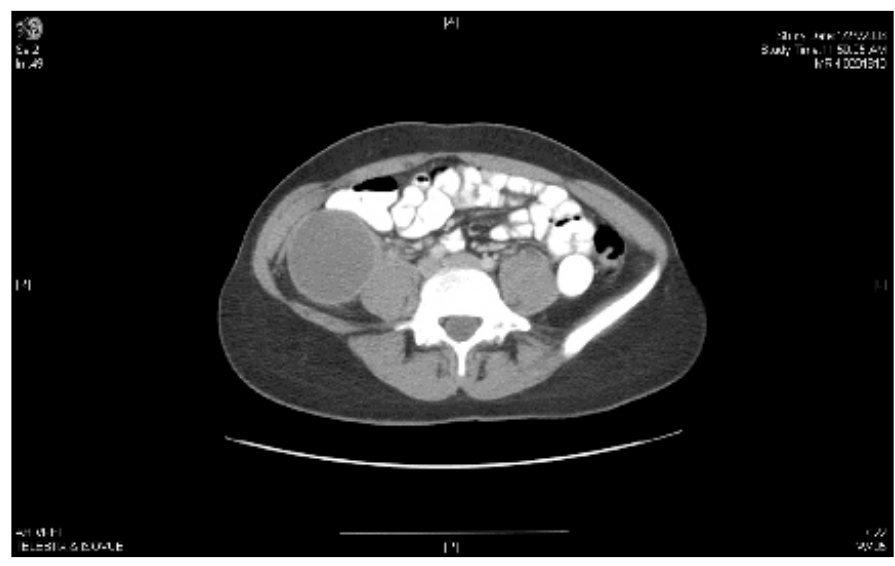

Fig. 2. A computed tomography scan showing the 6-cm thick-walled retroperitoneal cystic mass.

minate nature of the lesion and the fact that the patient was symptomatic, surgical excision was recommended rather than diagnostic cyst aspiration. The patient, a young female, wanted to minimize abdominal scarring so a laparoscopic approach was undertaken with the understanding that the cystic lesion would be treated as a malignancy and conversion to laparotomy would ensue if there were any concerns regarding the completeness of excision or cyst fluid spillage intraoperatively. Fortunately, there were no intraoperative problems and the cyst remained intact without any spillage during dissection, aspiration and extraction. Aspiration of the cyst fluid allowed the specimen to be extracted through one of the $10 \mathrm{~mm}$ laparoscopic ports, thus obviating the need for a larger extraction incision.

\section{Conclusion}

Our case is unique because it represents a case of cystic retroperitoneal endometriosis that had converted to ade-

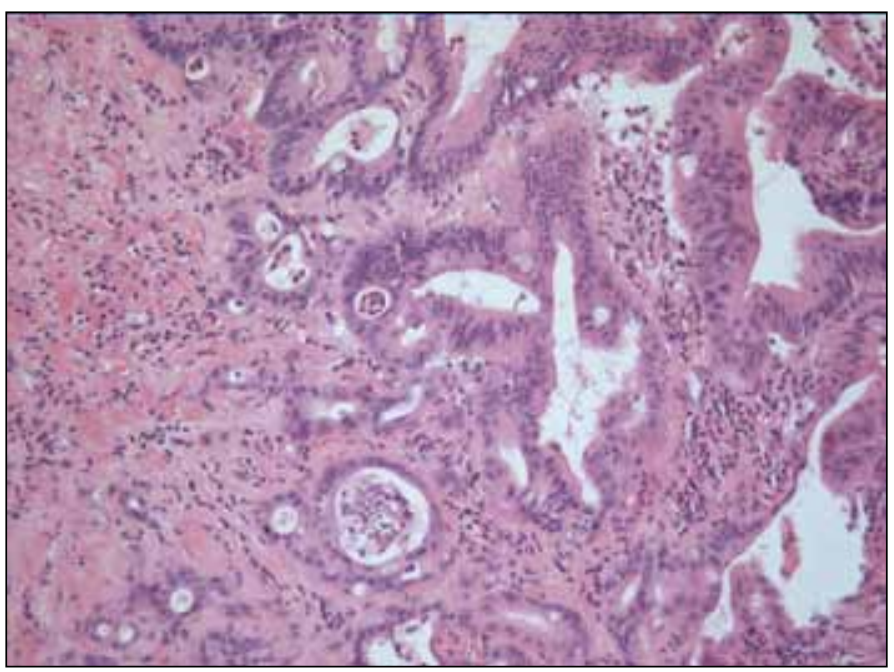

Fig. 3b. Histology showing adenocarcinoma arising in the wall of the cyst. 
nocarcinoma. Furthermore, the neoplasm was adequately treated with laparoscopic monotherapy No further treatment has been required, as the patient is well after 3 years of follow-up. Particularly in younger, symptomatic patients, retroperitoneal cystic lesions should be excised and treated as being malignant until proven otherwise.

*Assistant Professor, Department of Urology, Queen's University, Kingston General Hospital, Kingston, ON; 'Associate Professor, Department of Obstetrics/Gynaecology, Queen's University, Kingston General Hospital, Kingston, ON; ${ }^{\ddagger}$ Assistant Professor, Department of Laboratory Medicine, Division of Pathology, University of Toronto, St. Michael's Hospital, Toronto, ON; §Associate Professor, Division of Urology, University of Toronto, St. Michael's Hospital, Toronto, ON

Competing interests: None declared.

This paper has been peer-reviewed.

\section{References}

1. Fishman A, Demirel D, Laucirica R, et al. Malignant tumours arising in endometriosis: clinical-pathological study and flow cytometry analysis. Eur I Obstet Gynecol Reprod Biol 1996;70:69-74.

2. Griffith $L M$, Carcangiu ML. Sex cord tumour with annular tubules associated with endometriosis of the fallopian tube. Am J Clin Pathol 1991;96:259-62.

3. Viganò P, Parazzini F, Somigliana E, et al. Endometriosis: epidemiology and aetiological factors. Best Pract Res Clin Obstet Gynaecol 2004; 18:177-200.

4. Benoit L, Arnould L, Cheynel N, et al. Malignant extraovarian endometriosis: a review. Eur I Surg Oncol 2006;32:6-11.

5. Paillocher N, Pessaux P, Catala L, et al. Malignant tumours arising in extra-ovarian endometriosis: a case report [in French]. J Gynecol Obstet Biol Reprod (Paris) 2005;34:501-3.

6. Leiserowitz GS, Gumbs IL, Oi R, et al. Endometriosis-related malignancies. Int I Gynecol Cancer 2003; 13:466-71.

7. Ness RB. Endometriosis and ovarian cancer: thoughts on shared pathophysiology. Am J Obstet Gynecol 2003;189:280-94

Correspondence: Dr. Darren Beiko, Assistant Professor, Department of Urology, Queen's University, Kingston General Hospital, 76 Stuart St., Kingston, ON K7L 2V7; fax: 613-548-2498; beikod@ kgh.kari.net 